\title{
Parámetros genéticos relacionados con características del fruto en berenjena (Solanum melongena L.)
}

\section{Genetic parameters related to fruit characteristics in eggplant (Solanum melongena L.)}

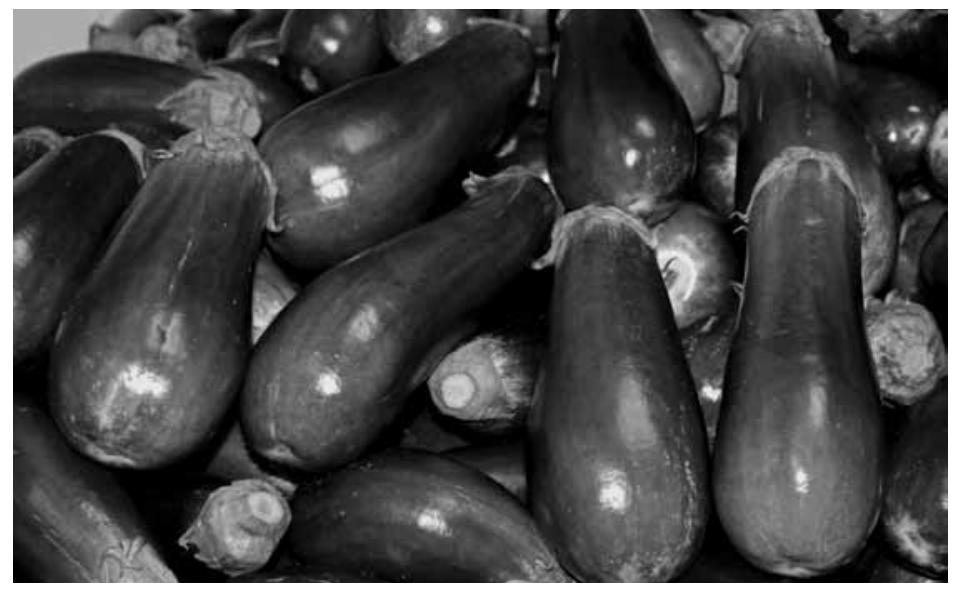

HERMMES ARAMÉNDIZ-TATIS1,2

CARLOS CARDONA-AYALA ${ }^{1}$

CÉSAR VERGARA-CÓRDOBA ${ }^{1}$

Frutos de berenjena.

Foto: J.A. Cantero

\section{RESUMEN}

El departamento de Córdoba es el principal productor de berenjena en el Caribe colombiano, pero el fruto de esta hortaliza presenta amplia variación en la forma y tamaño que disminuye la calidad exigida por el mercado y su competitividad. El objetivo de estudio fue determinar los parámetros genéticos del fruto relacionados con la forma, firmeza, color predominante, diámetro y longitud, peso de semillas y número de semillas. Se evaluaron 36 líneas de berenjena y tres testigos comerciales, bajo el diseño de bloques aumentados de Federer, con nueve bloques y unidades experimentales de dos surcos de $10 \mathrm{~m}$, con distancias entre surcos y plantas de $1 \mathrm{~m}$. El análisis de varianza reveló diferencias significativas y altamente significativas para todas las características estudiadas, entre testigos, líneas y en el contraste líneas versus testigos, revelando con ello la existencia de variabilidad genética. El coeficiente de variación genético para todos los caracteres fluctuó entre 7,33\% y $42,79 \%$ y la heredabilidad entre $60,45 \%$ y $89,97 \%$, calificada como moderada. El avance genético resultó alto para firmeza del fruto, número de semillas por futo y peso total de semillas por fruto, por lo que métodos de selección simple pueden modificar la estructura de la población.

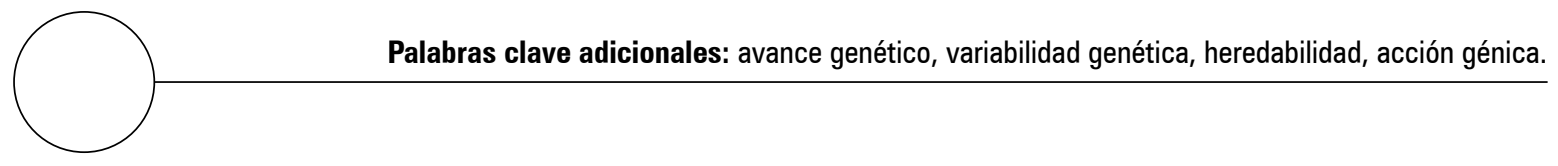

Facultad de Ciencias Agrícolas, Universidad de Córdoba, Montería (Colombia).

Autor para correspondencia.haramendiz@hotmail.com 


\section{ABSTRACT}

The department of Córdoba is the principal producer of eggplant in the Colombian Caribbean, but the fruit of this vegetable presents wide variation in shape and size, which decreases the quality required by the market and its competitiveness. The aim of this study was to determine the genetic parameters related to fruit shape, fruit firmness, predominant fruit color, diameter and fruit length, weight of seeds per fruit and number of seeds per fruit. 36 lines of eggplant and three commercial controls, under a design of augmented blocks with nine blocks and experimental units of two rows of $10 \mathrm{~m}$ with distances between rows and plants of $1 \mathrm{~m}$, were evaluated. Analysis of variance revealed significant and highly significant differences for all the studied characteristics, between the controls, the lines and, the contrast between controls and lines, revealing the existence of genetic variability. The coefficient of genetic variation ranged between $7.33 \%$ and $42.79 \%$ for all traits, and between $60.45 \%$ and $89.97 \%$ for heritability (moderate). The genetic advance was high for fruit firmness, number of seeds per fruit and weight of seeds per fruit, for which simple selection methods can modify the structure of the population.

Additional key words: genetic gain, genetic variability, heritability, gene action.

Fecha de recepción: 31-03-2014

Aprobado para publicación: 03-06-2014

INTRODUCCIÓN

La berenjena es una hortaliza popular en el Caribe húmedo colombiano $y$, según las últimas estadísticas disponibles en Agronet (2014), en el año 2006, el departamento de Córdoba es el principal productor con 131 ha, que representan el 46,13\% del área nacional, una producción de 689 t y un rendimiento de 5,3 $\mathrm{t} \mathrm{ha}{ }^{-1}$; sustentada en la siembra de variedades locales como Criolla lila, Criolla negra, Criolla morada, Criolla blanca, las cuales han surgido como resultado de mutaciones, hibridación natural y selección realizada por el hombre en las poblaciones heterogéneas homocigotas, que aún conservan características no deseables, como plantas altas, presencia de espinas en las hojas y cáliz, cojines florales hasta de cinco flores, frutos de tamaño, color y forma variable, características que inciden fuertemente en la calidad y rendimiento; por tanto, existe una mezcla de genotipos y como resultado, baja calidad del producto en el mercado (Araméndiz et al., 2008).

La situación mundial en cuanto al consumo de alimentos ha cambiado y cada día se demandan productos agrícolas de mejor calidad, como conse- cuencia de un mayor conocimiento de los consumidores de la relación entre una buena dieta y la salud. En este sentido, fueron exportadas 439.304 $t$ de berenjena por valor de US\$468.018.000 (FAO, 2014), por ser una de las 10 hortalizas con demanda creciente por parte de los consumidores de productos saludables, dado su bajo poder calórico $(24 \mathrm{kcal} / 100 \mathrm{~g})$ y propiedades antioxidantes (eliminación de radicales libres) y propiedades reductoras del colesterol (Bletsos, 2002; Islam et al., 2010) y obesidad (Prabhu et al., 2010).

La estimación de parámetros genéticos para caracteres agronómicos han sido reportados en diferentes especies agrícolas por diversos investigadores (Muniappan et al., 2010; Kumar et al., 2013), lo cual ha permitido cambios de gran importancia en caracteres económicos y desarrollo de cultivares acordes a las necesidades del agricultor y consumidor final.

Por tanto, los programas de mejoramiento genético en una especie como la berenjena, con un amplio potencial de consumo a nivel mundial, 
debe considerar las preferencias y gustos del consumidor en cuanto a forma, tamaño, color y número de semillas en el fruto (Mendonça et al., 2005). En este sentido, la estimación de la variabilidad genética en cualquier población es fundamental para conocer la respuesta a la selección así como el progreso genético. Por lo tanto, la identificación de parámetros genéticos como: varianza genética, coeficiente de variación genético y heredabilidad entre otros, es de gran importancia, ya que a través de ellos se puede conocer la magnitud de la variabilidad genética y posibilidades de progreso a través de la selección directa e indirecta (Rocha et al., 2003; Kumar et al., 2013).

El objetivo del presente trabajo fue estimar los parámetros genéticos y verificar la existencia de variación genética para características de fruto y posibilidades de progreso a través de la selección en cultivares criollos de berenjena existentes en la región Caribe colombiana.

\section{MATERIALES Y MÉTODOS}

\section{Localización}

El experimento se realizó en el segundo semestre de 2012 en el municipio de Sampués (Sucre, Colombia), finca La Florida, cuyas coordenadas geográficas corresponde a 9¹0' 59" latitud norte y $75^{\circ} 22^{\prime} 54^{\prime \prime}$ longitud oeste. El área de estudio registró una temperatura promedio de $27,9^{\circ} \mathrm{C}$, una precipitación de $1.100 \mathrm{~mm}$, humedad relativa promedio del $88 \%$, brillo solar de $1.860 \mathrm{~h}$ año-1 y suelo de textura franco arcilloso-arenoso.

\section{Material experimental}

Para el estudio se evaluaron 36 líneas obtenidas por selección individual en cultivares criollos, destacadas por poseer características de color de fruto lila, negro y púrpura, así como forma de fruto alargada y tres testigos comerciales: Long Purple, Anasac y Galine.

\section{Diseño experimental}

Se utilizó el diseño experimental de bloques aumentados propuesto por Federer (1961), con nueve bloques y unidades experimentales de dos surcos de $10 \mathrm{~m}$, con distancias entre surcos y plantas de $1 \mathrm{~m}$, para 20 plantas por unidad experimental, 140 plantas por bloque, y 1.260 plantas en los nueve bloques aumentados.

\section{Manejo agronómico}

El ensayo se estableció en un lote de $3.000 \mathrm{~m}^{2}$, se trazaron los bloques de tal manera que quedaran perpendiculares a un gradiente de conductividad hidráulica y se instaló riego por aspersión para atender los requerimientos hídricos.

Las plántulas fueron producidas en bolsas de polietileno y contenedores plásticos. El sustrato estuvo compuesto de una mezcla de bovinaza, arena y cascarilla de arroz en proporciones de 5:2:1 respectivamente; tratada con Trichoderma en dosis de $3 \mathrm{~g} \mathrm{~L}^{-1}$ de agua, al momento de la siembra de las semillas. Así mismo, al momento del trasplante a las respectivas unidades experimentales para reducir los riesgos fitosanitarios. El manejo de arvenses, plagas y enfermedades según manejo del agricultor y se acompañó de buenas prácticas agronómicas.

\section{Variables dependientes y registro de datos}

Las variables consideradas en el estudio fueron: forma del fruto (FORF), en la sexta semana de cosecha se describió la forma a cinco frutos tomados al azar en cinco plantas. Para ello se utilizó el descriptor para berenjena (IBPGR, 1990); firmeza del fruto (FF), en la décima semana de cosecha se midió la firmeza a tres frutos tomados al azar en cada tratamiento, con un penetrómetro digital FHT-803 (General Tools MFG, New York, NY); tomando dos lecturas por fruto, en la parte central en dos de las caras opuestas del mismo, la unidad de medida fue $\mathrm{kgf} / \mathrm{cm}^{2}$; color predominante del 
fruto (CPFRU), en cada cosecha se describió el color de cinco frutos tomados al azar. Para valorar el color, se utilizó la escala propuesta en el descriptor para berenjena (IBPGR, 1990); diámetro (DL) y longitud del fruto (LF), en la tercera cosecha se tomaron cinco frutos al azar, a los cuales se les determinó el diámetro en la parte central utilizando un nonio y a los mismos con ayuda de un metro se midió la longitud polar del fruto en centímetros; peso total semillas fruto (PTSF) y número de semillas por fruto (NSF), en la décima segunda de cosecha se seleccionaron e identificaron tres frutos al azar que se dejaron madurar en la planta y posteriormente fueron cosechados, para extraer, cuantificar y pesar el número de semillas de cada fruto, en gramos.

\section{Análisis de datos}

Con la información registrada en campo se realizaron análisis de varianza (Anova), contrastes líneas vs. testigos, así como entre testigos y entre líneas, medias ajustadas por cuadrados mínimos y errores estándar con el programa SAS versión 9.2 (SAS, 2008). Así mismo, fueron estimadas las varianzas fenotípica, genética, ambiental, heredabilidad en sentido amplio, coeficiente de variación genética en porcentaje y índice de variabilidad $b$, mediante el uso del programa GENES versión Windows (2004.2.1), desarrollado por Cruz (2004).

Heredabilidad en sentido amplio

$h_{a}^{2}=\frac{\sigma_{g}^{2}}{\sigma_{p}^{2}} \times 100$

La variabilidad $h_{a}^{2}$ en sentido amplio fue clasificada: baja (<30\%); moderada (30\% - 60\%) y alta (>60\%).

Coeficiente de variación genética

$C V_{g}=\frac{\sqrt{\sigma_{g}^{2}}}{\bar{x}} \times 100$
Índice de variabilidad $\quad b=\frac{C V_{g}}{C V_{a}}$

Avance genético $\left(\Delta_{g}\right)=\mathrm{k} \times \sigma_{p} \times h_{a}^{2}$

Donde:

$\mathrm{k}=$ Diferencial de selección al 5\% de intensidad de selección, equivalente a 2,06

$\sigma_{p}=$ Desviación estándar fenotípica

$h_{a}^{2}=$ Heredabilidad en sentido amplio

El avance genético $\left(\triangle_{g}\right)$ en porcentaje:

$\frac{\left(\Delta_{g}\right)}{\bar{x}} \times 100$

El rango del avance genético fue clasificado: bajo $(<10 \%)$, moderado $(10-20 \%)$ y alto $(>20 \%)$.

\section{RESULTADOS Y DISCUSIÓN}

Los resultados del análisis de varianza (tabla 1) indican que los cuadrados medios para los testigos, líneas y contrastes entre testigos y líneas, acusaron significancia entre las medias de tales fuentes de variación $(P \leq 0,01$ y $P \leq 0,05)$, indicando la existencia de diferencias genéticas en el germoplasma bajo estudio y posibilidades de mejoramiento por el aprovechamiento de los efectos genéticos aditivos y no aditivos, a través de la selección de plantas con atributos de fruto favorables, hecho que coincide con lo manifestado por Muñoz-Falcón et al. (2009), Muniappan et al. (2010), Kumar et al. (2013) y Toppino et al. (2013), para quienes esta especie posee una amplia variabilidad genética en los cultivares locales.

Para los caracteres evaluados se registró buena precisión experimental $(\mathrm{CV}<16 \%)$ con excepción de NSF y PTSF (tabla 1), lo que se traduce en alta confiabilidad de los valores fenotípicos en representar los valores genotípicos como lo sostienen Da Silva et al. (1999); Araméndiz et al. (2011). 
Tabla 1. Cuadrados medios del análisis de varianza para cinco características de calidad de fruto en berenjena (Solanum melongena L.)

\begin{tabular}{|l|c|c|c|c|c|c|}
\hline Fuente & $\mathrm{GL}$ & $\mathrm{LF}$ & $\mathrm{DF}$ & $\mathrm{FF}$ & $\mathrm{NSF}$ & $\mathrm{PTSF}$ \\
\hline Bloques & 8 & 6,77 & 0,31 & 33,90 & $398.213,98$ & 4,50 \\
\hline Testigos & 2 & $2,33^{*}$ & $0,37^{* *}$ & $148,14^{* *}$ & $326.411,00^{* *}$ & $3,73^{* *}$ \\
\hline Líneas & 35 & $102,83^{* *}$ & $64,38^{* *}$ & $6,45^{* *}$ & $284.702,00^{* *}$ & $4,17^{* *}$ \\
\hline T vs. L & 1 & $175,25^{* *}$ & $14,36^{* *}$ & $15,86^{*}$ & $7.053 .983,18^{* *}$ & $107,72^{* *}$ \\
\hline Error & 16 & 1,00 & 0,10 & 2,94 & $41.401,18$ & 0,44 \\
\hline Total & 62 & 8,59 & 2,58 & 13,81 & $347.091,00$ & 4,67 \\
\hline$x$ & & 17,82 & 6,16 & 10,74 & 1104,67 & 3,52 \\
\hline CV (\%) & & 5,61 & 5,13 & 15,97 & 18,41 & 18,86 \\
\hline$R^{2}$ & & 0,96 & 0,98 & 0,94 & 0,96 & 0,97 \\
\hline
\end{tabular}

$x=$ media, CV $(\%)=$ coeficiente de variación $\mathrm{GL}=$ grados de libertad, NS $=$ no significativo, ${ }^{*}=$ significativo al $5 \%,{ }^{*}{ }^{*}=$ significativo al $1 \%$, $\mathrm{GL}=$ grados de libertad, $\mathrm{LF}=$ longitud del fruto, $\mathrm{FF}=$ firmeza del fruto, $\mathrm{DF}=$ diámetro del fruto, NSF = número de semillas por fruto, $\mathrm{PTSF}=$ peso total de semillas por fruto.

Los altos valores pueden obedecer a fallas en la polinización, debido principalmente a las altas temperaturas $\left(>35^{\circ} \mathrm{C}\right)$, que afectan la superficie estigmática y los granos de polen (Yan et al., 2011; Araméndiz et al., 2012) y así mismo, alta humedad relativa restringe la movilidad del polen (Pessarakli y Dris, 2004); por lo que las condiciones ambientales influyen en la producción de semilla.

El valor medio registrado para LF fue de $17,82 \pm 0,12 \mathrm{~cm}$; para los testigos $15,89 \pm 0,33 \mathrm{~cm}$ y en las líneas 19,26 $\pm 1,13$ (tabla 2), destacándose los cultivares CO 22, $\mathrm{PH} 28$ y SO 06, con 22,66; 22,42 y $22,06 \mathrm{~cm}$, respectivamente. Esto posibilita la selección de plantas de frutos de mayor longitud que junto a los diámetros registrados, corresponden al tipo serpentiforme, que favorece el corte en rodajas a lo largo de toda la longitud y permite aumentar el rendimiento de elaboración para la agroindustria.

Los estimadores de la varianza genética $(1,99)$ con respecto a la varianza fenotípica $(2,99)$; coeficiente de variación genético $(7,33 \%)$ e índice de variabilidad genética $b(1,41)$ corroboran la existencia de variabilidad genética y alta heredabilidad (66,64\%), para un progreso moderado del
$12,20 \%$ (tabla 2), lo que posiblemente obedece a efectos genéticos no aditivos que están influyendo en la reducida ganancia como lo sostienen Prabhu et al. (2009).

La media del DF fue de $6,16 \pm 0,03 \mathrm{~cm}$; para los testigos 5,61 $\pm 0,33 \mathrm{~cm}$ y en las líneas de 6,58 $\pm 0,36$ $\mathrm{cm}$ (tabla 2), sobresaliendo las líneas $\mathrm{CO} 38 ; \mathrm{PH}$ 09 y $\mathrm{PH} 24$, que acusaron valores de 5,98; 5,61 y $4,95 \mathrm{~cm}$, respectivamente. En este sentido, Macua et al. (2005), indican que diámetros entre 3,0 y 6,0 $\mathrm{cm}$, son ideales para la agroindustria.

La magnitud de la varianza genética $(0,32)$ resultó superior con respecto a la varianza ambiental $(0,10)$ lo que indica que este carácter cumple un papel importante en el rendimiento del fruto (Reddy et al., 2013). Así mismo, el coeficiente de varianza genético $(8,64 \%)$ e índice de variabilidad b $(1,79)$ favorecen la alta heredabilidad $(76,36 \%)$ y ganancia genética moderada del 15,49\% (tabla 2). Estos resultados concuerdan con los reportados por Pirinç y Pakyürek (2004), Prabhu et al. (2009) y Kumar et al. (2013), quienes anotan que la presencia de genes con acción aditiva y no aditiva, explican el porqué de una alta heredabilidad y reducido progreso genético, 


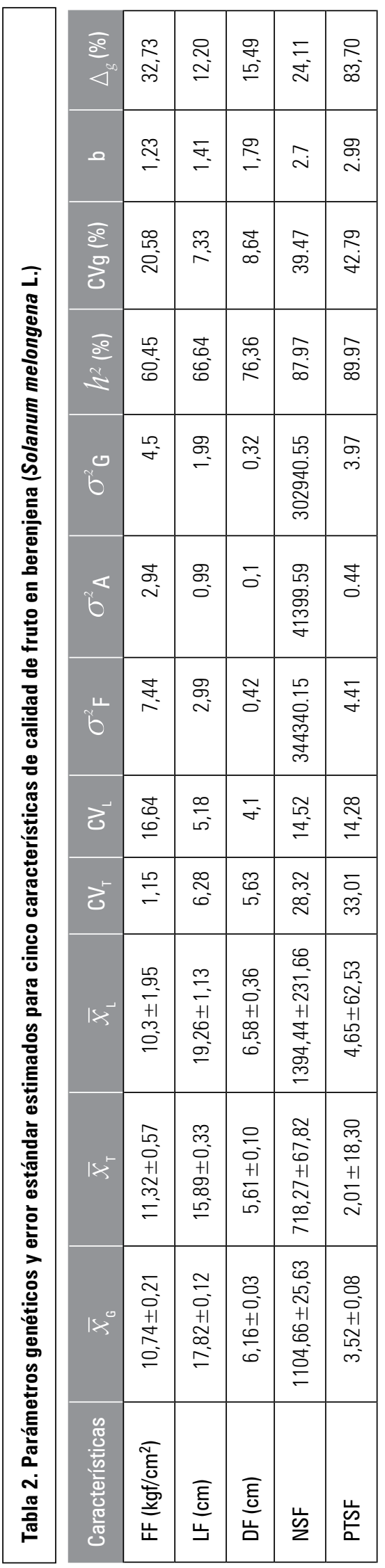

dado que la varianza genética de dominancia no es aprovechable en el mejoramiento poblacional, limitando la obtención de frutos de mayor diámetro (Mendoça et al., 2005).

El promedio general de NSF fue de 1.104 $\pm 25,63$ semillas; para los testigos $718,27 \pm 67,82$ semillas y en las líneas 1394,44 $\pm 231,66$ semillas (tabla 2), destacándose las líneas P06 (781), PH 17 (711), C015 (667), C010 (659) y PH 10 (603), lo que permite una mayor producción de biomasa, lo cual es concordante con los reportados por Sankar et al. (2001). Esta superioridad de las líneas puede obedecer a diferencias en las cantidades de polen depositadas sobre cada estigma y su incidencia en el crecimiento, desarrollo de frutos y semillas (Polverente et al., 2005; Cardoso, 2005), ya que en los cultivares testigos es posible que las altas temperaturas $\left(>35^{\circ} \mathrm{C}\right)$ generan tétradas anormales durante la meiosis y los granos de polen no germinan (Yan et al., 2011).

La estimación de la varianza genética $(302.940,55)$ resultó superior a la varianza ambiental $(41.399,59)$ y el coeficiente de variación genética $(39,47 \%)$ e índice de variabilidad b $(2,7)$ denotan el potencial de avance favorable en este carácter que asociados con la alta heredabilidad $(87,97 \%)$ y alta ganancia $(24,11 \%)$ en la selección de cultivares con menor cantidad de semillas, sustentan la acción génica aditiva que controla la característica analizada (Reddy et al., 2013).

En cuanto al PTSF el promedio general fue de $3,52 \pm 0,08 \mathrm{~g}$, en los testigos $2,01 \pm 0,22 \mathrm{~g}$ y en las líneas 4,65 $\pm 0,75 \mathrm{~g}$ (tabla 2) valores cercanos a los reportados por Passam et al. (2010). Las diferencias con relación a los testigos posiblemente sea reflejo tanto de la cantidad de polen depositado en las superficies estigmáticas como del número de óvulos presentes en el gineceo, lo que a su vez podría influir en la calidad fisiológica de las semillas por la competencia por fotoasimilados, conduciendo a un menor tamaño de la semilla y por consiguiente, de su embrión (Cardoso, 2005). En esta característica sobresalen los 
cultivares PH 28 (3,71); C010 (3,34), P 09 (3,03), C015 (2,75), P06 (2,52), PH $17(2,01)$ y $\mathrm{PH} 10$ $(1,55)$, siendo los dos últimos genotipos ideales para el consumo en fresco.

La diferencia entre la varianza fenotípica $(4,41)$ y la varianza genética $(3,97)$ resultó estrecha, indicando que el genotipo contribuyó mucho a la expresión del PTSF, lo que sugiere mayores posibilidades de mejora a través de la selección, puesto que el coeficiente de variación genética (42,79\%) e índice de variabilidad b $(2,99)$, explican un alto avance genético (83,70\%), en la reducción del número de semilla, dada la heredabilidad (89,97\%) encontrada donde genes con posible acción génica aditiva sean los principales responsables de su herencia y métodos simples de selección permitan dicho propósito, lo cual concuerda con lo reportado por Ansari et al. (2011).

La FF del fruto registró una media general de $10,74 \pm 0,21 \mathrm{kgf} / \mathrm{cm}^{2}$, mientras en los testigos se registraron valores de $11,32 \pm 0,57 \mathrm{kgf} / \mathrm{cm}^{2}$ y en las líneas de 10,30 $\pm 1,95 \mathrm{kgf} / \mathrm{cm}^{2}$ (tabla 2), denotando con ello superioridad de los testigos, especialmente el híbrido Galine que registró 15,51 kgf/ $\mathrm{cm}^{2}$, frente al promedio de los genotipos promisorios sometidos a evaluación. Sin embargo, se encontraron genotipos con ventaja agronómica en este carácter importante para el mercado y para la satisfacción del consumidor como el PH 18 (14,02 $\left.\mathrm{kgf} / \mathrm{cm}^{2}\right)$, P04 $\left(14,35 \mathrm{kgf} / \mathrm{cm}^{2}\right)$, P03 (14,92 kgf/ $\mathrm{cm} 2)$ y PH $23\left(18,52 \mathrm{kgf} / \mathrm{cm}^{2}\right)$; corroborando la existencia de diferencias genéticas entre los genotipos evaluados. Como fue reportado en otros cultivares por Cybulska et al. (2011). Estos valores superan los registros de $5,36 \mathrm{kgf} / \mathrm{cm}^{2}$, reportados por Gajewski y Arasimowicz (2004), quienes además anotan que este carácter está asociado con el momento de cosecha, variaciones en la morfología celular, propiedades químicas (cantidad de pectina) y mecánicas de la pared celular, que inciden en la textura de la fruta (Rouphael et al., 2010; Trad et al., 2014) y así mismo, con la minimización de las pérdidas de agua, de sustancias internas y del daño mecánico (Lara et al., 2014).
La varianza genética $(4,5)$ resultó de superior magnitud que el componente ambiental $(2,94)$, lo cual se refleja en la alta heredabilidad (60,45\%), coeficiente de variación genético $(20,58 \%)$ e índice de variabilidad $b(1,23)$, que permitieron un avance del $32,73 \%$, lo que puede obedecer a genes con acción génica aditiva (Prabhu et al., 2009). En este sentido, Da Silva et al. (1999) resaltan predominancia de los efectos genéticos aditivos y es posible, que ello refleje alta heredabilidad en poblaciones con nulo mejoramiento genético.

A excepción del híbrido Galine, que registró forma aperada y un color de fruto negro, los otros cultivares acusaron colores lila, púrpura y negro y forma alargada, cuya herencia es de naturaleza poligénica con acción génica aditiva y no aditiva (Qiao et al., 2011), apetecida en el mercado local, nacional y mundial. Además, la coloración púrpura, debida a las antocianinas localizadas en las vacuolas de las células, sugiere un fuerte poder reductor que probablemente desempeña un papel importante como antioxidante y en la regulación del estado redox de la célula (Concellón et al., 2007; Muñoz-Falcón et al., 2009), está bajo el control genético de tres genes con acción génica complementaria (Makani et al., 2007).

\section{CONCLUSIONES}

Las 36 líneas de berenjena presentan variabilidad genética y heredabilidad en sentido amplio, en los parámetros relacionados con la calidad de fruto y posibilitan la obtención de ganancias importantes a través de la selección, especialmente en el número de semillas por fruto y peso total de semillas por fruto.

\section{AGRADECIMIENTOS}

A la Universidad de Córdoba por la financiación del proyecto "Evaluación de la heterosis y habilidad combinatoria de la berenjena (Solanum melongena L.)". 


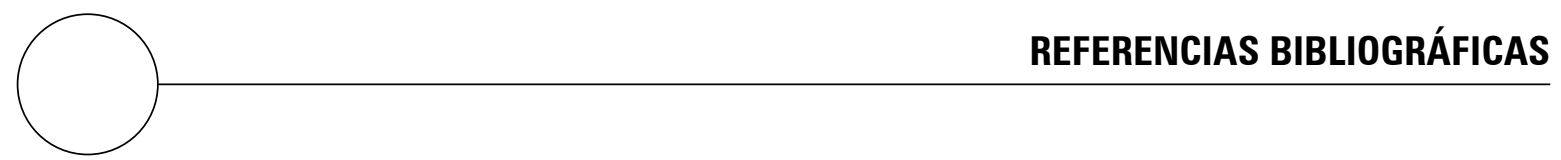

Agronet. 2006. Análisis estadístico de área y producción agrícola y pecuaria. En: http://www.agronet.gov. co; consulta: febrero de 2014.

Ansari, S.F., N. Mehta, S. Ansari y J.P. Gavel. 2011. Variability studies in Brinjal (Solanum melongena L.) in Chhattisgarh plains. Electr. J. Plant Breed. 2(2), 275-281.

Araméndiz-Tatis. H., A.C. Cardona, O.A. Jarma y CM. Espitia. 2008. El cultivo de la berenjena (Solanum melongena L.). Produmedios, Bogotá.

Araméndiz-Tatis, H., C. Cardona y E. Lugo. 2012. Germinación del polen de berenjena (Solanum melongena L.) en condiciones in vitro. Rev. Fac. Nac. Agron. Medellin 65(2), 6643-6649.

Araméndiz-Tatis, H., C.P. Sudré, L.S.A. Gonçalves y R. Rodrigues. 2011. Potencial agronômico e divergência genética entre genótipos de berinjela nas condições do Caribe Colombiano. Hortic. Bras. 29(2), 174-180.

Bletsos, F.A. 2002. Evaluation of new and commercial eggplant hybrids and cultivars in relation to their frozen product. Acta Hort. 579, 89-93.

Cardoso, A.I.I.2005. Polinização manual em abobrinha: efeitos nas produções de frutos e de sementes. Hortic. Bras. 23(3), 731-734.

Concellón, A., M. Añón y A. Chaves. 2007. Effect of low temperature storage on physical and physiological characteristics of eggplant fruit (Solanum melongena L.). LWT 40 (3), 389-396.

Cruz, C.D. 2004. Programa genes versão windows: aplicativo computacional em genética e estatística. Universidade Federal Viçosa, Viçosa, Brasil.

Cybulska, J., A. Zdunek y K. Konstankiewicz. 2011. Calcium effect on mechanical properties of model cell walls and apple tissue. J. Food Eng. 102(3), 217-223.

Da Silva, D.J.H., C.P. Da Costa, V. Casali, L.A. Dias y C.D. Cruz. 1999. Análise da capacidade combinatória em berinjela. Bragantia 58(1), 7-14.

FAO. 2014. Cultivos y produtos de ganadería. www.fao. org; consulta: febrero de 2014.

Federer, W.T. 1961. Augmented designs with one-way elimination of heterogeneity. Biometrics 17, 447-473

Gajewski, M. y D. Arasimowicz. 2004. Sensory quality of eggplant fruits (Solanum melongena L.) as affec- ted by cultivar and maturity stage. Pol. J. Food Nutr. Sci. 13/54(3), 249-254.

IBPGR. 1990. Descriptor for eggplant. International Board for Plant Genetic Resources, Roma.

Islam, S., H. Tomoko, K. Ishikawa, N. Takeda, M. Azad y K. Miyouchi. 2010. Growth and fruit quality responses of hydroponically cultivated eggplants to mineral controlled deep sea water. J. Plant Nutr. 33(13), 1970-1979.

Kumar, S.R., T. Arumugan, S. Balakrishnan y C.R. Anandakumar. 2013. Variability in the segregating generation of eggplant for earliness and yield. Pak. J. Biol. 16(20), 1122-1129.

Lara, I., B. Belge y L.F. Goulao. 2014. The fruit cuticle as a modulator of postharvest quality. Postharv. Biol. Technol. 87,103-112.

Macua, J.I., I. Lahoz, S. Calvillo y A. Santos. 2005. Berenjena. Variedades con destino industrial. Navarra Agraria 149, 39-42.

Makani, J.M., B.A. Monpara y H.R. Dhameliya. 2007. Inheritance of certain traits in brinjal (Solanum melongena L.). Natl. J. Plant Improv. 9(1), 32-35.

Mendonça, R., F. Mangan y M. Moreira. 2005. Evaluation of eggplant (Solanum spp.) varieties for ethnic groups in the United States. Proc. Interamer. Soc. Trop. Hort. 48, 38-39.

Muniappan, S., K. Saravanan y B. Ramya. 2010. Studies on genetic divergence and variability for certain economic characters in eggplant (Solanum melongena L.). Electr. J. Plant Breed. 1(4), 462-465.

Muñoz-Falcón, J.E., J. Prohens, S. Vilanova y F. Nuez. 2009. Diversity in commercial varieties and landraces of black eggplants and implications for broadening the breeders' gene pool. Ann. Appl. Biol. 154(3), 453-465.

Passam, H.C., S. Theodoropoulou, T. Karanissa e I.C. Karapanos. 2010. Influence of harvest time and after-ripening on the seed quality of eggplant. Sci. Hortic. 125(3), 518-520.

Pessarakli, M.M. y R. Dris. 2004. Pollination and breeding of eggplants. Food Agr. Environ. 2(1), 218-219.

Pirinç, V. y A.Y. Pakyürek. 2004. A study on comparison of eggplant population with their selfing lines. Intl. J. Agr. Biol. 6 (5), 874-876. 
Polverente, M.R., D. Carneiro y A.I. Cardoso. 2005. Produção e qualidade de sementes de berinjela em função do horário de polinização manual. Bragantia 64(3), 467-472.

Prabhu, M., S. Natarajan y L. Pugalendhi. 2009. Variability and heritability studies in F5 and F6 progenies of brinjal (Solanum melongena L.). Amer.-Eurasian J. Sustain. Agric. 3(3), 306-309.

Prabhu, M., S. Praneetha y S. Natarajan. 2010. Organoleptic evaluation of brinjal genotypes. Agric. Sci. Digest. 30(1), 73-74.

Qiao, J., F. Liu, Y. Chen y Y. Lian. 2011. Study on inheritance of eggplant fruit shape. Acta Hort. 38(11), 2121-2130.

Reddy, B.P.K., H. Begun, N. Sunil y M.T. Reddy. 2013. Variance component analysis of quantitative traits in muskmelon (Cucumis melo L.). Trakia J. Sci. 11(2),118-124

Rocha, M. de M., J.E. Campelo, F.R. Freire Filho, V.O. Ribeiro y A.C. Almeida. 2003. Estimativas de parâmetros genéticos em genótipos de caupí de tegumento branco. Rev. Cient. Rural 8(1), 135 141.
Rouphael, Y., D. Schwarz, A. Krumbein y G. Colla. 2010. Impact of grafting on product quality of fruit vegetables. Sci. Hortic. 127(2), 172-179.

Sankar, V., P. Jansirani, V.A. Sathiyamurthy y D. Veeraragavathatham. 2001. Studies on the efficient seed production technology in brinjal F1 hybrid ' $\mathrm{COBH} 1$ ' (EP 45 x CO 2). Capsicum Eggplant Newsl. 20, 106-109.

SAS. 2008. User's guide SAS/STAT® version 9.2. SAS Institute, Cary, NC.

Toppino, L., L. Barchi, G.L. Rotino, G. Vale, N. Acciarri, T. Ciriaci, E. Portis y S. Lanteri. 2013. Mapping of QTLs for key breeding traits in eggplant (Solanum melongena L.). pp. 177-884. Proc. XV Meeting on Genetics and Breeding of Capsicum and Eggplant EUCARPIA. 2-4 Sept. de 2013. Torino, Italia.

Trad, M., C. Ginies, B. Gaaliche, C. Renard y M. Mars. 2014. Relationship between pollination and cell wall properties in common fig fruit. Phytochem. 98(1), 78-84.

Yan, L., L. Ling, C. Xiu-de, Y. Bao-xing y G. Dongsheng. 2011. Effects of high temperature on the anther and pollen development of sweet cherry in solar greenhouse. Acta Hort. 38(6), 1029-1036. 